\title{
Structure of Synthetic Transmembrane Lipid Membranes at the Solid/Liquid Interface Studied by Specular X-ray Reflectivity
}

\author{
Thomas Schubert, ${ }^{\dagger}$ Peter C. Seitz, ${ }^{\dagger}$ Emanuel Schneck, ${ }^{\dagger}$ Makoto Nakamura,, \\ Motonari Shibakami, ${ }^{\ddagger}$ Sergio S. Funari, ${ }^{\S}$ Oleg Konovalov," and Motomu Tanaka, ${ }^{* \dagger}$ \\ Biophysical Chemistry II, Institute of Physical Chemistry and BIOQUANT, University of Heidelberg, INF 253, \\ D-69120 Heidelberg, Germany, Institute of Biological Resources and Functions, National Institute of Advanced \\ Industrial Science and Technology, Tsukuba, Japan, HASYLAB at DESY, Notkestr. 85, D-22603 Hamburg, \\ Germany, and European Synchrotron Radiation Facility (ESRF), 38053 Grenoble, Cedex 9, France
}

Received: May 5, 2008; Revised Manuscript Received: May 26, 2008

\begin{abstract}
We fabricated a new class of supported membranes based on monolayers of artificial bola (transmembrane) lipids. The lipids used in this study are symmetric bola lipids with two phosphocholine head groups, which resemble natural archaea lipids. To prevent bending of the hydrocarbon chains, stiff triple bonds are inserted in the middle of the hydrocarbon cores. The formation of homogeneous "monolayers" of transmembrane lipids over macroscopic areas can be monitored with fluorescence microscopy. Structures of such supported monolayers in bulk water were characterized with specular X-ray reflectivity using high energy X-ray radiation, which guarantees a high transmission through bulk water. Here, the vertical structure of single monolayers could be resolved from reconstructed electron density profiles. To verify the structural model suggested by the specular reflectivity, we also performed small- and wide-angle X-ray scattering of transmembrane lipid suspensions. The wide-angle patterns reflect a distorted chain-chain correlation, while the small-angle scattering allowed us to model an electron density profile which is consistent with the profile calculated from specular reflectivity.
\end{abstract}

\section{Introduction}

Archaebacteria or simply archaea are considered a distinct domain of life next to eucaryota and bacteria. They can be found in extreme habitats such as hot springs or hypersaline water, and they are extremely abundant in the environment. ${ }^{1}$ The most remarkable differences between archaea and other cells exist in the cell membrane composed of unique transmembrane lipids, whose physicochemical characteristics have been studied by many research groups. ${ }^{2-6}$ On the other hand, several types of transmembrane lipids and bola amphiphiles have synthetically been designed to mimic the key structural motifs of the unique archaeal membranes. ${ }^{7,8}$ Previously, Patwardhan et al. reported the synthesis of artificial transmembrane lipids with diacetylenes but with no control of the stereochemistry of the ether junctions. ${ }^{7}$ As reported in our recent account, ${ }^{9}$ we succeeded in the control of the chirality in the ether junctions by efficient protection/ deprotection procedures, which could be used as a more realistic model system for archaeal membranes.

Among various biomembrane models, planar lipid membranes deposited on solid supports (so-called supported membranes ${ }^{10}$ ) enable us to fully characterize the structure and functions of the membranes using various physical techniques. In this study, we deposited monolayers of transmembrane lipids on glass substrates and silicon wafers by incubating with membrane suspensions. Here, we used two types of transmembrane lipids (Figure 1) that contain two phosphocholine head groups (as in the native transmembrane motif isocaldarchaeol). In contrast

* To whom correspondence should be addressed. Email: tanaka@ uni-heidelberg.de.

$\dagger$ University of Heidelberg.

\$ National Institute of Advanced Industrial Science and Technology.

$\S$ HASYLAB at DESY.

"European Synchrotron Radiation Facility.

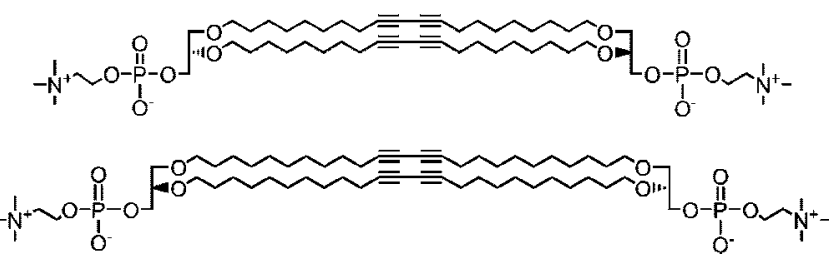

Figure 1. Structures of C20PCPC (top) and C24PCPC (bottom) symmetric cyclic lipids with phosphocholine head groups and stabilizing diacetylene bonds in the core.

to natural caldarchaeols with branched and saturated hydrocarbon chains, each hydrophobic chain contains a rigid diacetylene in the middle, which was reported as the key structural moiety to avoid bending of the backbone into a U-shape. ${ }^{11}$ After removing the unbound membranes by intensive rinsing, the homogeneity of the supported membrane was checked with fluorescence microscopy, and the lateral fluidity of the membrane was determined by fluorescence after photobleaching (FRAP). To resolve the structure of the supported membrane in bulk water, we used specular X-ray reflectivity with high energy X-ray radiation $(22 \mathrm{keV})$, which guarantees approximately $40 \%$ transmission through $1 \mathrm{~cm}$ of water. As recently demonstrated by Miller et al., ${ }^{12}$ this technique can be used as an alternative to the commonly used specular neutron reflectivity ${ }^{13}$ for characterizing biological membranes at the solid/ liquid interface with a much higher throughput rate and a higher spatial resolution. However, previous studies merely dealt with well-characterized standard lipids, ${ }^{12,14,15}$ and thus, the application of such a technique to more unusual molecular constructs is still missing. As a complementary technique to verify the specular reflectivity results, we conducted small- and wide-angle X-ray scattering (SAXS and WAXS) experiments of transmem- 
brane lipid suspensions and calculated the electron density profile perpendicular to the membrane surface.

\section{Experimental Section}

Chemicals. For all aqueous solutions, water from a Millipore purification system $(R>18 \mathrm{M} \Omega \mathrm{cm}$, Millipore, Molsheim, France) was used. Buffer used was phosphate buffered saline (PBS) at pH 8 with $10 \mathrm{mM} \mathrm{NaH} \mathrm{PO}_{4}$ and $100 \mathrm{mM} \mathrm{NaCl}$. If not stated otherwise, chemicals were purchased from SigmaAldrich (Munich, Germany) and used without further purification.

Preparation of Supported Membranes. For microscopy and FRAP measurements, substrates were standard microscope coverslips $\left(24 \times 24 \mathrm{~mm}^{2}\right)$, while for X-ray reflectivity experiments silicon wafers with native oxide were used as mentioned in the main text. They were cleaned by ultrasonication and rinsing in acetone, ethanol, and methanol and subsequently immersed in a solution of 1:1:5 (v/v/v) $\mathrm{H}_{2} \mathrm{O}_{2}(30 \%) / \mathrm{NH}_{4} \mathrm{OH}$ $(30 \%) /$ water at $60{ }^{\circ} \mathrm{C}$ for $30 \mathrm{~min}$. Finally, they were rinsed intensively with water and dried at $70{ }^{\circ} \mathrm{C}$. To prepare lipid vesicle suspensions, lipids were dried into thin films either under a stream of nitrogen or with a rotary evaporator and stored in vacuum overnight. After suspending the lipids, the suspension incubated at $70{ }^{\circ} \mathrm{C}$ for more than $2 \mathrm{~h}$. The resulting vesicle suspension was then incubated on the glass or silicon substrates at $70{ }^{\circ} \mathrm{C}$ for at least $1 \mathrm{~h}$. Afterward, the samples were vigorously rinsed with buffer.

Fluorescence Microscopy and FRAP. Fluorescence images were taken on a standard inverted microscope Axiovert 200 (Zeiss, Oberkochen). Fluorescence recovery after photobleaching (FRAP) was measured with a self-built setup described previously. ${ }^{16,17}$ In brief, a strong laser pulse $(\sim 700 \mathrm{~mW}$, for 200-500 ms) through a pinhole is used to bleach the sample in a circular spot with a diameter of $9.3 \mu \mathrm{m}$. The recovery of the fluorescence signal by lateral diffusive exchange of bleached by unbleached lipids in the fluid lipid matrix is observed with an attenuated beam $(\times 1000)$ on a photomultiplier, and the data are fitted according to the theory of Soumpasis et al. ${ }^{18,19}$ This allows the extraction of the diffusion coefficient $D$ in $\mu \mathrm{m}^{2} / \mathrm{s}$ and the mobile fraction.

Specular Reflectivity at the Solid/Liquid Interface and SAXS/WAXS in Suspension. Specular reflectivity measurements were performed at the ID10B beamline at the European Synchrotron Radiation Facility (ESRF, Grenoble, France) by illuminating the samples at $22 \mathrm{keV}$. SAXS/WAXS experiments were conducted at the A2 beamline at HASYLAB (DESY, Hamburg, Germany) using a photon energy of $8 \mathrm{keV}$. Cyclic lipid suspensions with a concentration of $20 \mathrm{wt} \%$ were filled into quartz capillaries (Hilgenberg, Malsfeld, Germany), centrifuged, and flame-sealed. Measurements were performed after conducting several heating/cooling cycles to cancel the thermal history of each sample.

\section{Results and Discussion}

Fluorescence Microscopy and FRAP. After sample preparation as outlined above, the formation of homogeneous membranes of C24PCPC (Figure 2A) and C20PCPC (Figure 2B) on a glass slide could be confirmed with fluorescence microscopy. Although uniform coverage of the whole glass slide could be observed with no remarkable defects, the fluorescence recovery after photobleaching (FRAP) results on both membranes showed no sign of diffusion at $20{ }^{\circ} \mathrm{C}$ (Figure 2C). On the other hand, the lack of lateral diffusion cannot be explained by polymerization of diacetylene units, since ${ }^{1} \mathrm{H}$ NMR and UV/

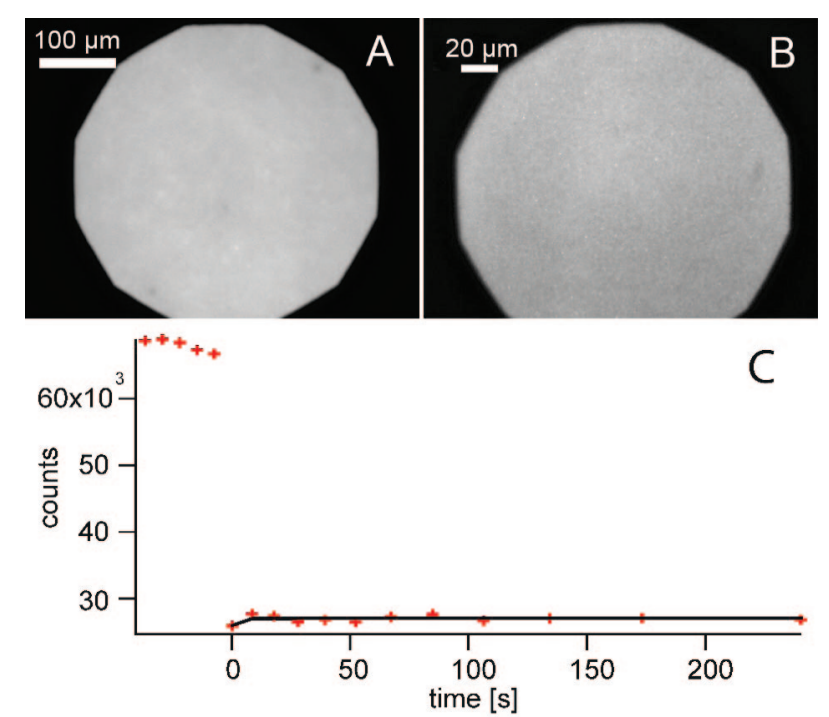

Figure 2. Fluorescence images of a (A) C24PCPC membrane and (B) C20PCPC membrane with $0.1 \mathrm{~mol} \%$ Texas Red-DHPE, deposited on glass. (C) Fluorescence recovery after photobleaching (FRAP) curve for $\mathrm{C} 24 \mathrm{PCPC}$ at $20^{\circ} \mathrm{C}$, showing no sign of lateral diffusion.

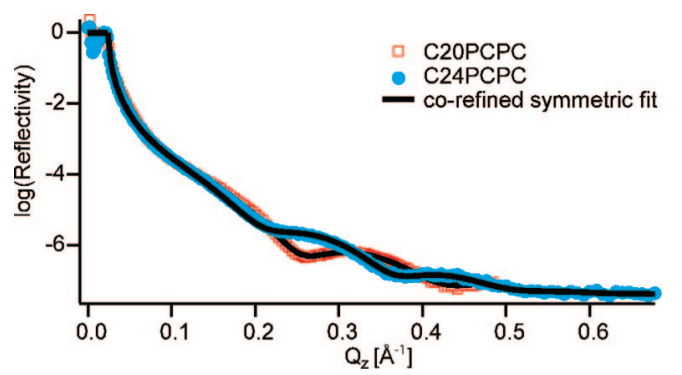

Figure 3. Specular reflectivity curves (markers) and the best fit results (solid lines) of C20PCPC (red squares) and C24PCPC (blue circles).

vis absorption spectra showed no change after exposing the samples to more than $10 \mathrm{~h}$ of daylight.

Specular Reflectivity of Supported Membranes at the Solid/Liquid Interface. Figure 3 represents the specular X-ray reflectivities of C20PCPC (red squares) and C24PCPC (blue circles). Here, the membranes were deposited on silicon wafers covered with native oxide (SiMat, Landsberg am Lech, Germany) in a self-built liquid cell. Both curves exhibit two pronounced minima, indicating the presence of layers with high electron density contrasts. The fact that the first and second minima of C24PCPC appear at lower $q$-values than those of C20PCPC further indicates that C24PCPC forms thicker layers than C20PCPC.

To fit the measured reflectivity curves, we model the supported membranes with three slabs, consisting of two slabs of head groups (layers 1 and 3) that sandwich one slab of hydrocarbon chains (layer 2). Here, we use the Parratt formalism $^{20}$ with a genetic minimization algorithm implemented in the Motofit software package. ${ }^{21}$ Motofit also enables us to fit several data sets simultaneously and to link parameters during fitting, both within and across data sets. Owing to the unique structural symmetry of the transmembrane lipids with rigid diacetylene cores, several parameters can be linked during the fitting. This is in contrast to normal phospholipid bilayers that consist of two independent (and thus uncorrelated) monolayer leaflets. Such an approach is especially powerful, as it reduces the number of free parameters and helps to perform the modeling without choosing arbitrary constraints. For instance, the slabs 
TABLE 1: Best Results Obtained with the Co-refined Symmetrical Fit to C20PCPC and C24PCPC Data

\begin{tabular}{lcccl}
\hline & layers & $D[\AA]$ & SLD $\left[\times 10^{-6} \AA^{-2}\right]$ & $\sigma[\AA]$ \\
\hline C20PCPC & 1 & $10.0^{a}$ & 10.4 & $2.6^{b, c}$ \\
& 2 & 16.7 & 8.0 & $2.0^{d}$ \\
& 3 & 10.0 & 10.4 & 2.0 \\
C24PCPC & 1 & 10.0 & & \\
& 2 & 22.9 & 10.4 & 2.6 \\
& 3 & 10.0 & 10.4 & 4.3
\end{tabular}

${ }^{a}$ See text for which elements are linked. ${ }^{b}$ Note that the roughness of layer 1 was not linked. ${ }^{c}$ Roughness values are defined from the side of bulk water toward the given layer. Note that the roughness at the interface between the head group and bulk $\mathrm{Si}$ was $3.3 \AA$. ${ }^{d}$ The lowest limit of the rms roughness is limited to be $\sigma \geq$ $2.0 \AA$ А.

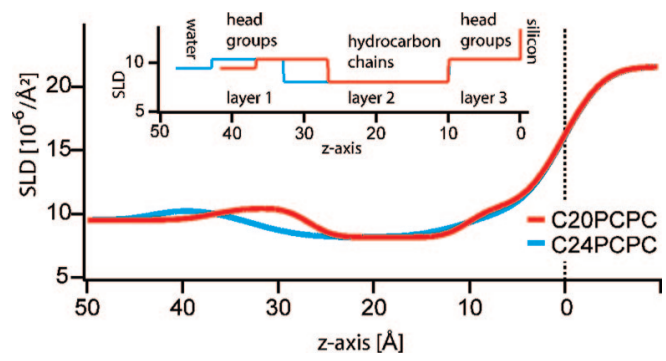

Figure 4. Scattering length density (SLD) profiles for C20PCPC and C24PCPC, determined with co-refined symmetric models. A slab model without roughness is shown in the inset.

of head groups are required to be consistent for both the inner leaflet (in contact to the solid substrates) and the outer leaflet (in contact with bulk water).

The best fit results we achieved with the co-refined, symmetric slab model are presented as solid lines in Figure 3. The links and constraints used were as follows: (i) the thickness and scattering length density (SLD) of the head groups (layers 1 and 3) are linked within and between data sets, (ii) the SLD of hydrocarbon chains (layer 2) is linked between both data sets, (iii) the interface roughness below and above the chain region are linked within each data set, and (iv) both background and bulk silicon/water parameters are set constant for both data sets.

The thickness $d$, scattering length density SLD $=r_{\mathrm{e}} \rho_{\mathrm{e}}$, and root mean square (rms) roughness $\sigma$ of individual slabs and interfaces are summarized in Table 1, where $r_{\mathrm{e}}$ is the Compton radius and $\rho_{\mathrm{e}}$ is the electron density. The corresponding SLD profiles of C20PCPC and C24PCPC are presented in Figure 4. It should be noted that both silicon oxide and the water gap between the substrate and the membrane cannot be clearly identified in both cases. The contrast between silicon and silicon oxide is low (see the Supporting Information), and the thin water gap is likely hidden in the interfacial roughness between head groups (layer 3 ) and silicon $(\sigma>3 \AA)$. The total thicknesses of C20PCPC and C24PCPC membranes are calculated to be 36.7 and $42.9 \AA$, respectively, indicating that both lipids form monolayers at the solid/liquid interface. The thickness difference $\Delta d \sim 6.2 \AA$ can reasonably be attributed to the difference in length of the hydrocarbon chains. The thickness of the head group regions (10 $\AA$ ) agrees well with previous reports on dimyristoylphosphatidylcholine (DMPC), ${ }^{22}$ while the scattering length densities of head groups $\left(10.4 \times 10^{-6} \AA^{-2}\right)$ and hydrocarbon chains $\left(8.0 \times 10^{-6} \AA^{-2}\right)$ are slightly lower than those of DMPC membranes in the $\mathrm{L}_{\beta^{\prime}}$ phase $\left(13 \times 10^{-6}\right.$ and $9.3 \times 10^{-6} \AA^{-2}$, respectively). Especially the fact that the electron density of the chain slab is lower than that of water

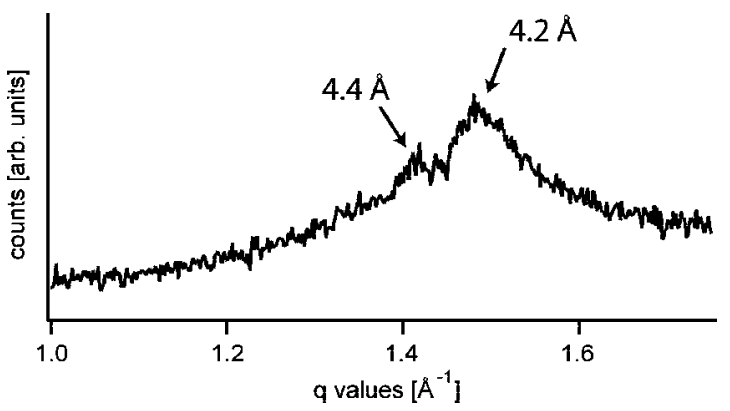

Figure 5. Wide-angle scattering data of $\mathrm{C} 24 \mathrm{PCPC}$ at $20{ }^{\circ} \mathrm{C}$. The correlation distances corresponding to the peak positions are indicated in the graph.

indicates that the penetration of water into the hydrocarbon core is negligible. On the other hand, the rms roughness values between the water and outer head groups $(\sigma=2.6 \AA)$ are even smaller than the $\sigma=6 \AA$ value reported for fluid phosphocholine membranes, ${ }^{12}$ indicating the formation of smooth, homogeneous membranes.

Small- and Wide-Angle X-ray Scattering of Vesicle Supensions. Initial X-ray scattering experiments of vesicle suspensions were performed for C24PCPC. Wide-angle X-ray scattering (WAXS) of C24PCPC measured at $20{ }^{\circ} \mathrm{C}$ (Figure 5) showed a chain-chain correlation peak corresponding to a correlation distance of $4.2 \AA$, accompanied by a satellite peak for a correlation at $4.4 \AA$. They can be observed up to $T=50$ ${ }^{\circ} \mathrm{C}$, consistent with the FRAP results that showed no sign of lateral fluidity at $20^{\circ} \mathrm{C}$ which be accounted for in terms of the ordering of hydrocarbon chains below the chain melting temperature (see the Supporting Information). The presence of a satellite peak at $4.4 \AA$ indicates defects or strains in the lateral chain packing, which is possibly due to a molecular tilt. In fact, it is plausible that that the chains cannot take all-trans conformation without significant strain or displacement of the molecule, if one considers the bond angles in the hydrocarbon chain region of a minimum energy model (see the Supporting Information). Further experiments such as grazing-incidence $\mathrm{X}$-ray diffraction at the solid/liquid interface will be helpful to gain deeper insights into the lateral structural ordering and thermotropic phase behavior of transmembrane lipid molecules including diacetylene moieties.

As presented in Figure 6A (black line), the SAXS signal of C24PCPC measured at $20{ }^{\circ} \mathrm{C}$ showed clear first and second order peaks, confirming that $\mathrm{C} 24 \mathrm{PCPC}$ forms ordered lamellar stacks. The membrane periodicity can be calculated from the peak position to be $4.9 \mathrm{~nm}$. To calculate scattering intensities from the form factor and structure factor, a self-developed analysis routine was used to analyze the SAXS results. Since the number of peaks observed in our experiments was small and thus the resolution of the model is limited, the conventional Fourier reconstruction fails. Therefore, we introduced a few reasonable constraints for the direct fitting of SAXS data. Similar to the case of specular reflectivity results, the C24PCPC monolayer was modeled with three slabs (head group, hydrocarbon chains, and head group), resulting in the model presented in Figure 6 and Table 2. The rms roughness at each slab interface and that of total membrane roughness are assumed to be about $2 \AA$. It should be noted that the height ratio between the first and second order peaks can be well explained by the suppression of the second order peak due to the overlap with a form factor minimum. In fact, the position of the form factor minima is significantly influenced by changing parameters such as the hydrocarbon slab electron density or head group position. As a 

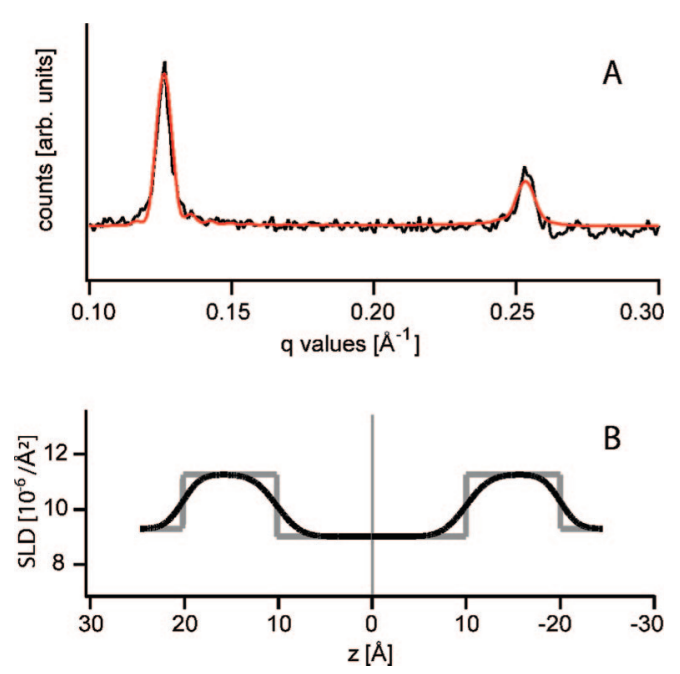

Figure 6. (A) Small-angle scattering results (black) and the best modeling result (red) of $\mathrm{C} 24 \mathrm{PCPC}$ at $20^{\circ} \mathrm{C}$, and (B) the corresponding electron density profile and the slabs.

TABLE 2: Parameters Corresponding to the Best Model for SAXS (See Figure 6) ${ }^{a}$

\begin{tabular}{ccc}
\hline C24PCPC & $d[\AA]$ & SLD $\left[\times 10^{-6} \AA^{-2}\right]$ \\
\hline head groups & 10.0 & 11.3 \\
hydrocarbon & 20.2 & 9.0
\end{tabular}

${ }^{a}$ Interfacial rms roughness was estimated to be about $2 \AA$.

result, the SAXS data could be well understood with our models despite the fact that we could obtain only two peaks due to the limit of the detector used for this series of measurements. Repeated cycles of fitting with few free parameters at a time resulted in models where no parameter could be significantly altered from the achieved minimum. The reconstructed electron density profile (Figure 6B) agreed well with the profile calculated from the supported membrane. The slightly higher electron density suggested by SAXS results can be understood in terms of self-healing of local membrane defects in the suspended membranes. These results confirm that the reflectivity experiments observed the formation of single monolayers on a solid substrate.

\section{Conclusions}

In this work, we characterized the structures of supported membranes of synthetic transmembrane lipids at the solid/liquid interface. Since the transmembrane lipids used in this study (C20PCPC and C24PCPC) display two phosphocholine head groups, they mimic key structural features of native archaea membranes, which possess excellent stability even under extreme conditions. The formation of smooth homogeneous membranes on solid supports can first be monitored by fluorescence microscopy. Using specular reflectivity with high energy synchrotron radiation, we were able to resolve the structure of single monolayers perpendicular to the membrane surface: the total thicknesses of C20PCPC and C24PCPC are 36.7 and $42.9 \AA$, respectively. The difference in hydrocarbon layer thickness between C24PCPC and C20PCPC (6.2 $\AA)$ is reasonable from the difference in the chain length. The electron density profiles reconstructed from the reflectivity data seem consistent with those of suspended multilamellar stacks of the same lipids, calculated from SAXS experiments. Despite an excellent homogeneity obtained by fluorescence microscopy, FRAP results suggested no sign of lateral diffusion for both lipids at $20^{\circ} \mathrm{C}$. This can be attributed to the short-range ordering between hydrocarbon chains, whose correlation distance of about $4.2 \AA$ could also be detected by WAXS experiments.

The obtained results indicate that supported membranes of transmembrane lipids as established here will not only be used as a new class of molecular constructs but also offer a physical model of how the "chemical link" between the proximal and distal leaflets influences the structures of supported membranes and the function of membrane proteins. It has also been demonstrated that specular X-ray reflectivity with high enery synchrotron radiation is a powerful alternative to commonly used specular neutron reflectivity to characterize even more complex biomembrane models (such as artificial and native membranes on polymer supports ${ }^{23-25}$ ) because of its capability to achieve a high throughput rate and spatial resolution.

Acknowledgment. We thank Alberto Zibetti, Thomas Kaindl, and Fernanda Rossetti for their assistance in experiments. We thank the ESRF and HASYLAB for the synchrotron beam times. This work was supported by the German Science Foundation (DFG Ta 259/6) and Fonds der Chemischen Industrie. M.S. is thankful for the support from New Energy and Industrial Technology Development Organization (NEDO), AIST, and Ministry of Education, Culture, Sports, Science and Technology.

Supporting Information Available: Reflectivity of blank wafers, a molecular model of C24PCPC, and further FRAP results. This material is available free of charge via the Internet at http://pubs.acs.org.

\section{References and Notes}

(1) Horikoshi, K., Grant, W. D. Eds. Extremophiles - Microbial Life in Extreme Environments; Plenum: New York, 1998.

(2) Nishihara, M.; Koga, Y. J. Biochem. 1987, 101, 997.

(3) Tomoaia-Cotisel, M.; Chifu, E.; Zsako, J.; Mocanu, A.; Quinn, P. J.; Kates, M. Chem. Phys. Lipids 1992, 63, 131.

(4) Untersteller, E.; Fritz, B.; Bleriot, Y.; Sinay, P. C. R. Acad. Sci., Ser. IIc: Chim. 1999, 2, 429.

(5) Mathai, J. C.; Sprott, G. D.; Zeidel, M. L. J. Biol. Chem. 2001, 276, 27266.

(6) Benvegnu, T.; Brard, M.; Plusquellec, D. Curr. Opin. Colloid Interface Sci. 2004, 8, 469.

(7) Patwardhan, A. P.; Thompson, D. H. Org. Lett. 1999, 1, 241.

(8) Menger, F. M.; Chen, X. Y. Tetrahedron Lett. 1996, 37, 323.

(9) Miyawaki, K.; Takagi, T.; Shibakami, M. Synlett 2002, 1326.

(10) Sackmann, E. Science 1996, 271, 43.

(11) Patwardhan, A. P.; Thompson, D. H. Langmuir 2000, 16, 10340.

(12) Miller, C. E.; Majewski, J.; Gog, T.; Kuhl, T. L. Phys. Rev. Lett. $2005,94$.

(13) Gutberlet, T., Katsaras, J. Eds. Lipid Bilayers: structure and interactions; Springer Verlag: Berlin, 2001. 74.

(14) Novakova, E.; Giewekemeyer, K.; Salditt, T. Phys. Rev. E 2006,

(15) Hochrein, M. B.; Reich, C.; Krause, B.; Radler, J. O.; Nickel, B. Langmuir 2006, 22, 538.

(16) Purrucker, O.; Fortig, A.; Jordan, R.; Sackmann, E.; Tanaka, M. Phys. Rev. Lett. 2007, 98, 078102.

(17) Tanaka, M.; Hermann, J.; Haase, I.; Fischer, M.; Boxer, S. G. Langmuir 2007, 23, 5638.

(18) Axelrod, D.; Koppel, D. E.; Schlessinger, J.; Elson, E.; Webb, W. W. Biophys. J. 1976, 16, 1055.

(19) Soumpasis, D. M. Biophys. J. 1983, 41, 95.

(20) Parratt, L. G. Phys. Rev. 1954, 95, 359.

(21) Nelson, A. J. Appl. Crystallogr. 2006, 39, 273.

(22) Tristram-Nagle, S.; Liu, Y. F.; Legleiter, J.; Nagle, J. F. Biophys. J. 2002, 83, 3324 .

(23) Tanaka, M.; Sackmann, E. Nature 2005, 437, 656.

(24) Rehfeldt, F.; Steitz, R.; Armes, S. P.; Von Klitzing, R.; Gast, A. P.; Tanaka, M. J. Phys. Chem. B 2006, 110, 9171.

(25) Wong, J. Y.; Majewski, J.; Seitz, M.; Park, C. K.; Israelachvili, J. N.; Smith, G. S. Biophys. J. 1999, 77, 1445.

JP803937M 